\title{
Transmission of malaria to mosquitoes blocked by bumped kinase inhibitors
}

\author{
Kayode K. Ojo, ${ }^{1}$ Claudia Pfander, ${ }^{2}$ Natascha R. Mueller, ${ }^{1}$ Charlotte Burstroem, ${ }^{2}$ Eric T. Larson, ${ }^{3}$ \\ Cassie M. Bryan, ${ }^{3}$ Anna M.W. Fox, ${ }^{1}$ Molly C. Reid, ${ }^{1}$ Steven M. Johnson, ${ }^{4}$ Ryan C. Murphy, ${ }^{4}$ \\ Mark Kennedy, ${ }^{5}$ Henning Mann, ${ }^{5}$ David J. Leibly, ${ }^{3}$ Stephen N. Hewitt, ${ }^{3}$ Christophe L.M.J. Verlinde, ${ }^{3}$ \\ Stefan Kappe, ${ }^{5}$ Ethan A. Merritt, ${ }^{3}$ Dustin J. Maly, ${ }^{4}$ Oliver Billker, ${ }^{2}$ and Wesley C. Van Voorhis ${ }^{1}$ \\ 1Division of Allergy and Infectious Diseases, Department of Medicine, University of Washington, Seattle, Washington, USA. 2Wellcome Trust Sanger Institute, \\ Hinxton, Cambridge, United Kingdom. ${ }^{3}$ Department of Biochemistry and ${ }^{4}$ Department of Chemistry, University of Washington, Seattle, Washington, USA. \\ ${ }^{5}$ Seattle Biomedical Research Institute, Seattle, Washington, USA.
}

\begin{abstract}
Effective control and eradication of malaria will require new tools to prevent transmission. Current antimalarial therapies targeting the asexual stage of Plasmodium do not prevent transmission of circulating gametocytes from infected humans to mosquitoes. Here, we describe a new class of transmission-blocking compounds, bumped kinase inhibitors (BKIs), which inhibit microgametocyte exflagellation. Oocyst formation and sporozoite production, necessary for transmission to mammals, were inhibited in mosquitoes fed on either BKI-1treated human blood or mice treated with BKI-1. BKIs are hypothesized to act via inhibition of Plasmodium calcium-dependent protein kinase 4 and predicted to have little activity against mammalian kinases. Our data show that BKIs do not inhibit proliferation of mammalian cell lines and are well tolerated in mice. Used in combination with drugs active against asexual stages of Plasmodium, BKIs could prove an important tool for malaria control and eradication.
\end{abstract}

\section{Introduction}

Malaria remains a major cause of morbidity, affecting 225 million people a year (1). Though great strides have been made in reducing mortality through improved chemotherapy, insecticide-treated bed nets, and vector control, an estimated 781,000 people continue to die of malaria every year (1). Similarly, development of completely effective vaccines or control of Anopheles mosquitoes with behavioral patterns that promote malaria transmission remains a formidable challenge. Studies have shown that the potential for malaria transmission remains high after administration of drugs targeting asexual stages, even when gametocyte prevalence and density appear low (2). This is a problem for malaria control because circulating gametocytes that are infectious to mosquitoes persist for weeks after therapy (3). Even the 2 drugs with antigametocyte activity, primaquine and artemisinin combination therapy (ACT), are imperfect at stopping malaria transmission to mosquitoes. Primaquine kills mature but not immature gametocytes, while ACT kills immature but not mature gametocytes (3). Longer primaquine treatment can kill all gametocytes, but leads to gastrointestinal intolerance in some individuals and hemolysis in patients with glucose-6-phosphate dehydrogenase deficiency (3). These issues emphasize the need for new transmission-blocking strategies that can aid in malaria eradication.

Malaria parasites alternate between life cycle stages in human and mosquito hosts. Switches between these stages require elaborately coordinated signal transduction. Changes in intracellular calcium levels affect the activity of a family of apicomplexan-specific kinases called calcium-dependent protein kinases (CDPKs) (4). CDPKs have been associated with invasion, gliding motility, and other secretory pathways (5). Plasmodium falciparum CDPK1 (PfCDPK1) may be critical in regulating the motor complex that

Conflict of interest: The authors have declared that no conflict of interest exists. Citation for this article: J Clin Invest. 2012;122(6):2301-2305. doi:10.1172/JCI61822. powers erythrocyte invasion (6), while PfCDPK5 was shown to be critical for egress of $P$. falciparum merozoites from erythrocytes (7). In this report, we describe bumped kinase inhibitors (BKIs) that block infection of mosquitoes by malaria parasites. These compounds selectively and potently inhibit CDPK4, which is required for exflagellation of Plasmodium berghei microgametes (8) and has recently been shown to be connected with induction of exflagellation in $P$. falciparum microgametes (9), before fusion with the macrogamete, to form a zygote. The zygote undergoes transitional ookinete and oocyst stages to mature into infective sporozoites that are injected into a mammalian host during the female mosquito blood meal. Blocking exflagellation through the selective inhibition of Plasmodium CDPK4 would be expected to interrupt malaria transmission without being toxic to humans (10).

\section{Results and Discussion}

We have previously demonstrated that the ATP-binding pockets of Toxoplasma gondii and Cryptosporidium parvum CDPK1 can be selectively targeted by BKIs with large aromatic moieties displayed from the 3 position of the pyrazolopyrimidine scaffold due to the anomalously small gatekeeper residues (glycine) present in these kinases. Selective inhibition of $\mathrm{Tg} / \mathrm{Cp}$ CDPK1 with BKIs leads to blockage of mammalian-host cell invasion $(11,12)$. PfCDPK4 has a serine at the gatekeeper position (Figure 1), smaller than the gatekeeper in almost all mammalian kinases, and an overall binding pocket that is very similar to those of TgCDPK1 and CpCDPK1. A number of compounds in our $\mathrm{Tg} / \mathrm{Cp}$ CDPK1 BKI library were found to inhibit recombinant PfCDPK4 (rPfCDPK4), the most potent being BKI-1 with an $\mathrm{IC}_{50}$ (concentration to inhibit $50 \%$ of enzyme activity) of $4 \mathrm{nM}$ (Table 1). However, not all compounds that are potent inhibitors of $\mathrm{Tg} / \mathrm{C} p \mathrm{CDPK} 1$ have comparable activity against $r P f C D P K 4$. Despite the overall structural similarities in the ATP-binding pockets of CDPKs, small differences in the size of the gatekeeper residue may have a large effect on inhibitor potency. 


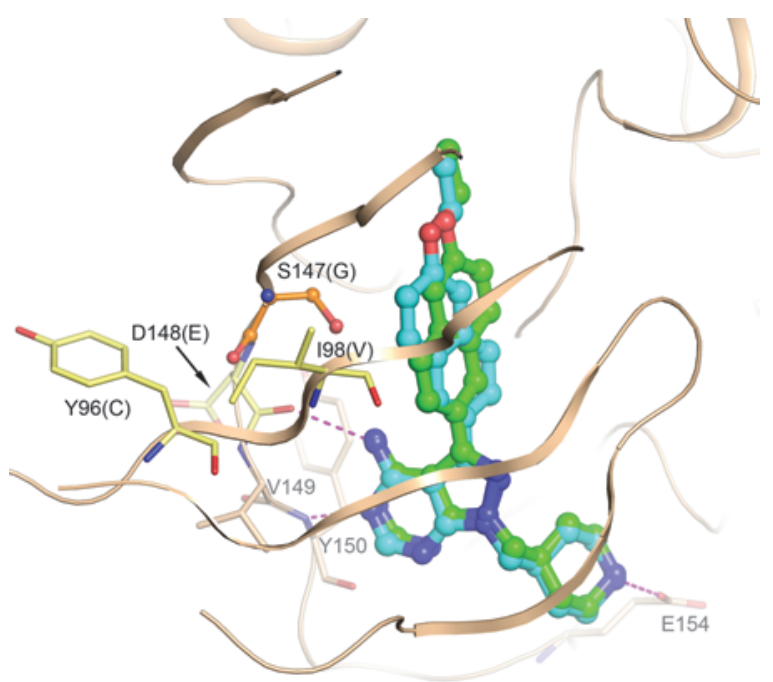

rPfCDPK1, which has a slightly larger threonine gatekeeper, was more than 20-fold less sensitive to BKI-1 than was rPfCDPK4 (Table 1). Furthermore, a rPfCDPK4 mutant (rPfCDPK4 S147M) that contains a methionine rather than a serine at the gatekeeper position was insensitive $\left(\mathrm{IC}_{50}>3000 \mathrm{nM}\right)$ to all of the BKIs tested (Table 1). BKI-1 was selected for further characterization because it is potent and selective for $\mathrm{rPfCDPK} 4$.

Cocrystal structures of BKIs complexed with TgCDPK1 are outstanding starting points for PfCDPK4 inhibitor modeling because the ATP-binding pocket of PfCDPK4 only differs from that of TgCDPK 1 by 4 residues (Figure 1). The most substantial difference is a serine gatekeeper in PfCDPK4 versus glycine in TgCDPK1; the 3 other substitutions are predicted to only contribute backbone atoms to the binding site. Two energetically favorable BKI-1-binding modes are predicted, differing by an approximately $180^{\circ}$ rotation of the ethoxynaphthyl "bump" relative to the scaffold (Figure 1). In both binding modes, the pyrazolopyrimidine scaffold maintained hydrogen bonds with the hinge region of the enzyme, which has been observed in multiple structures of TgCDPK1, but its predicted orientation relative to the pocket was rotated slightly

\section{Figure 1}

A homology model of PfCDPK4 was generated based on the crystal structure of the TgCDPK1:BKI-1 complex (PDB3sx9). Modeling suggests 2 possible binding modes for $\mathrm{BKI}-1$ in the active site of PfCDPK4, differing by the orientation of the "bump" in the "gatekeeper pocket," but very similar to the binding modes seen in the crystal structure with $T g C D P K 1$. Four residues within $8 \AA$ of the bound BKI differ between PfCDPK4 and TgCDPK1. These are labeled in black, with the $T g C D P K 1$ residue given in parentheses. Only the glycine/serine difference at the gatekeeper (orange sticks) is expected to have a significant influence on the binding mode of the inhibitor, as the other 3 (pale yellow sticks) contribute only backbone atoms to the pocket. Hydrogen bonds are depicted as magenta dashed lines. Conserved residues in the hinge region involved in binding and Glu154 are depicted as beige sticks and are labeled in gray. due to a necessary shift of the hydrophobic bump away from the side chain of the serine gatekeeper. Regardless of the orientation of the ethoxynaphthyl group, scaffold binding was not affected and the serine gatekeeper did not prevent high-affinity binding of BKI-1 to PfCDPK4. The methylpiperidine substituent of BKI-1 was predicted to make a hydrogen bond with Glu154, which has previously been observed in crystal structures of TgCDPK1 (11) in complex with $15 \mathrm{BKI}$ that contain this functional group.

BKI-1 potently inhibited the exflagellation of $P$. falciparum, but did not block the proliferation of asexual parasites (Table 1). This observation fits the hypothesis that BKI- 1 inhibits PfCDPK4 in vivo, and we sought chemical-genetic data to support this. Despite repeated attempts, we were unable to generate a drug-resistant S147M PbCDPK4 or PfCDPK4 mutant exogenously expressed in any Plasmodium species. Hence, we cannot at this time specify that BKI-1 blocks exflagellation and prevents Plasmodium infection of mosquitoes exclusively through the inhibition of CDPK4. In the absence of chemical-genetic evidence, we tested a series of BKIs in the enzymatic inhibition and cellular exflagellation assays to determine whether there is a correlation between these 2 activities.
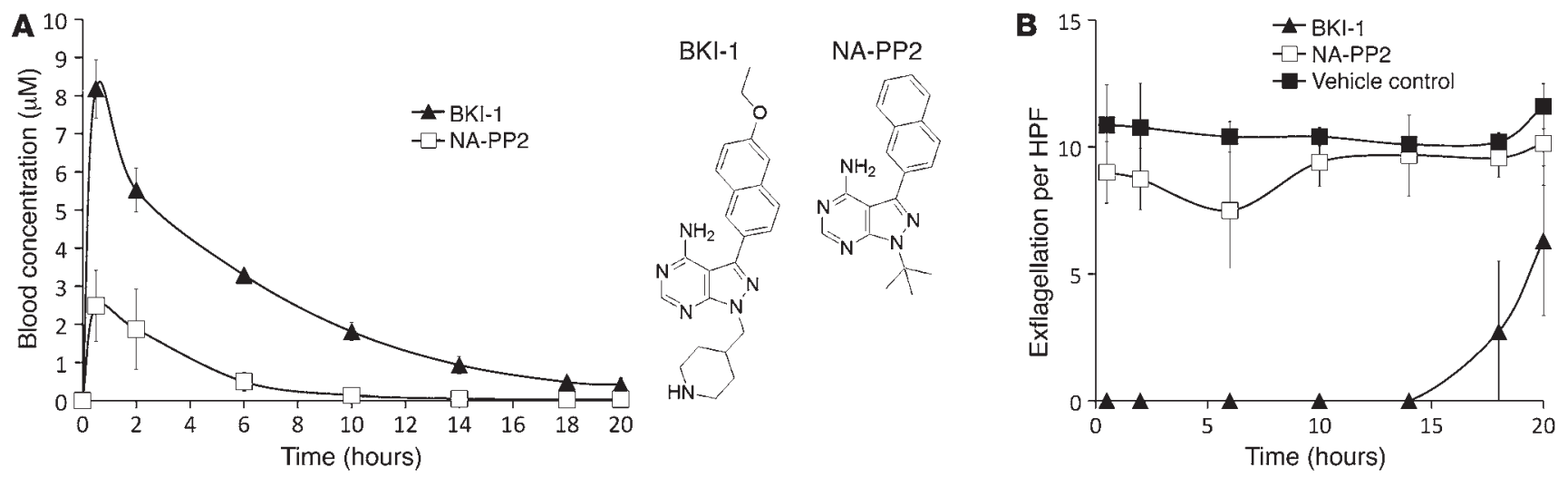

Figure 2

Plots show mouse blood BKI-1 or NA-PP2 concentration $(\mathbf{A})$ and the average number of exflagellation events per HPF (B) at varying times after $50 \mathrm{mg} / \mathrm{kg}$ administration i.p. to mice at time 0 . The structures of BKI-1 and NA-PP2 are shown in $\mathbf{A}$. There was complete suppression of male gametocyte differentiation to exflagellating units for 14 hours in the presence of BKI-1 relative to NA-PP2-treated or untreated controls. Data are representative of 3 experiments. Error bars represent SEM. 
Our data show a strong correlation between rPfCDPK4 inhibition and the blockage of exflagellation. BKIs with an $\mathrm{IC}_{50}$ of less than $50 \mathrm{nM}$ against rPfCDPK4 concurrently blocked exflagellation of $P$. falciparum microgametocytes with $\mathrm{EC}_{50}$ values of less than $300 \mathrm{nM}$ (Table 1 and Supplemental Table 1 for chemical structures; supplemental material available online with this article; doi:10.1172/ JCI61822DS1). Closely related analogs with a single modification that reduces their ability to inhibit rPfCDPK4 did not block exflagellation. Replacing the 1-(piperidin-4-ylmethyl) groups of BKI-1 ( IC $_{50}$ of $4 \mathrm{nM}$ ) and compound 1291 ( $\mathrm{IC}_{50}$ of $16 \mathrm{nM}$ ) with a 1-tert-butyl group resulted in analogs (1281 and NA-PP2) with reduced activities against $\mathrm{rPfCDPK} 4$ enzyme $\left(\mathrm{IC}_{50}=77.4\right.$ and 177 $\mathrm{nM}$, respectively). There was a corresponding reduction in the abilities of these compounds to block exflagellation, with 1281 and NA-PP2 demonstrating an $\mathrm{EC}_{50}$ of greater than $3000 \mathrm{nM}$ for exflagellation. Derivatives with variations at the 3 position of the pyrazolopyrimidine scaffold (for example, compound 1266) that greatly reduce $\mathrm{rPfCDPK} 4$ inhibition did not block exflagellation at the highest concentration tested. Therefore, analogs with substituents that are not well accommodated in the rPfCDPK4 hydrophobic gatekeeper pocket did not inhibit the catalytic activity of this enzyme and did not block exflagellation.

Targeted deletion of the $c d p k 4$ gene in P. berghei (P. berghei cdpk4 mutant) completely abrogates microgametocyte exflagellation in vitro and mosquito transmission in vivo (10). To determine whether a chemical inhibitor could have a similarly profound effect, a $P$. berghei $c d p k 4$ mutant was complemented with the Pfcdpk4 gene (Supplemental Figure 1, A-C). Expression of a myc epitope-tagged PfCDPK4 protein completely restored sexual development of P. berghei (Supplemental Figure 1D). Whether PbCDPK4 or PfCDPK4 were expressed, BKI-1 was equally effective at blocking exflagellation (Supplemental Figure 2), so WT P. berghei expressing GFP were used in subsequent experiments. Administration of $50 \mathrm{mg} / \mathrm{kg}$ BKI-1 i.p. resulted in a peak plasma concentration of $8.2 \mu \mathrm{M}$ and plasma levels exceeding $1 \mu \mathrm{M}$ for 14 hours after treatment (Figure 2A). When administered to P. berghei-infected mice at this dose, BKI-1 completely suppressed exflagellation in blood samples from treated mice 30 minutes after injection and up to 14 hours later (Figure 2B). However, 20 hours after dosing, when lower levels (0.41 $\mu \mathrm{M})$ of BKI-1 could be detected, some exflagellation was observed (Figure 2), demonstrating that the effect of BKI-1 is reversible.

To assess its impact on malaria transmission to mosquitoes, we injected P. berghei gametocyte-infected mice i.p. with $10 \mathrm{mg} / \mathrm{kg}$ BKI-1, a dose that has no impact on asexual parasitemia or gametocyte rates, but is sufficient to block exflagellation. Anopheles stephensi mosquitoes were allowed to feed on treated mice $30 \mathrm{~min}$ utes after dosing. P. berghei expressing GFP were used for ease of following oocyst production (Figure 3A). Treatment of mice at 10 $\mathrm{mg} / \mathrm{kg}$ completely blocked the formation of oocysts, while infected mice treated with vehicle or with control compound NA-PP2 gave rise to hundreds of oocysts per mosquito (Figure 3A). Similarly, mixing BKI-1 (final concentrations of $1 \mu \mathrm{M}$ or $3 \mu \mathrm{M}$ ) with human blood containing P. falciparum gametocytes prevented the exflagellation of microgametes and infection of $A$. stephensi midguts compared with vehicle control (Figure $3 \mathrm{~B}$ ). Furthermore, a complete absence of infective sporozoites was observed in dissected salivary glands of A. stephensi fed with $3 \mu \mathrm{M}$ BKI-1-treated blood (Figure $3 \mathrm{~B}$ ), which is consistent with the absence of exflagellation at this concentration. At a concentration of $100 \mathrm{nM}$, BKI-1 still reduced the prevalence of oocyst infections, and the sporozoite load in

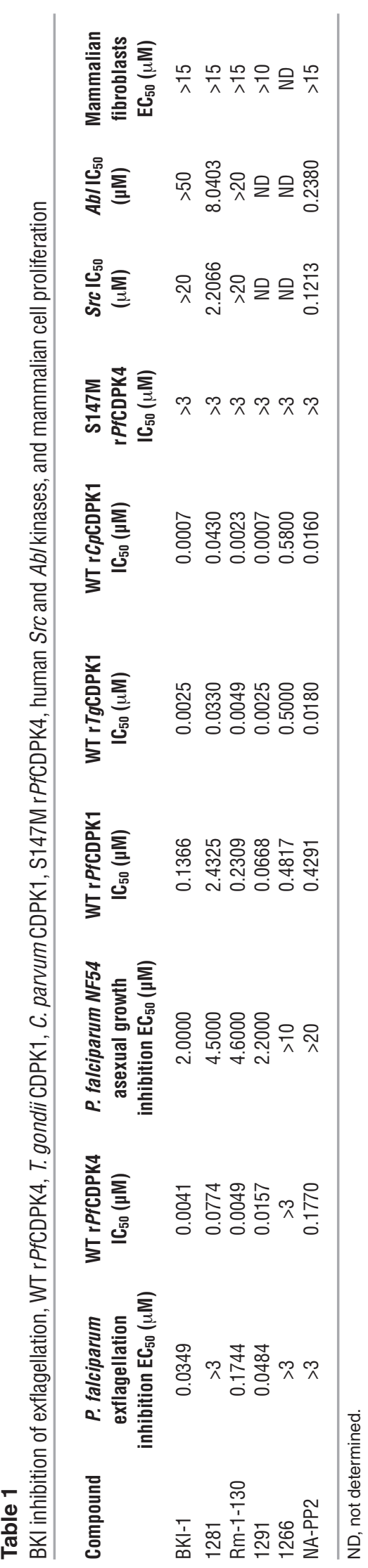


A

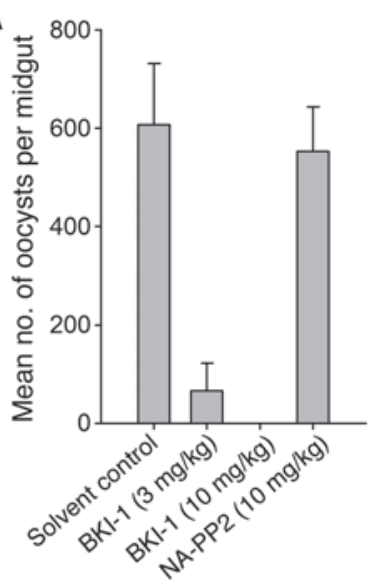

Solvent control

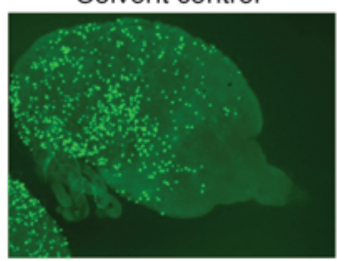

$\mathrm{BKI}-1$ treated $(10 \mathrm{mg} / \mathrm{kg})$

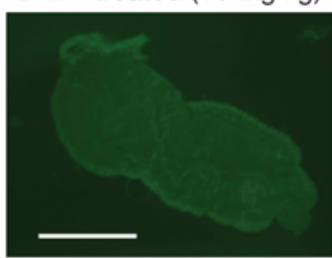

B
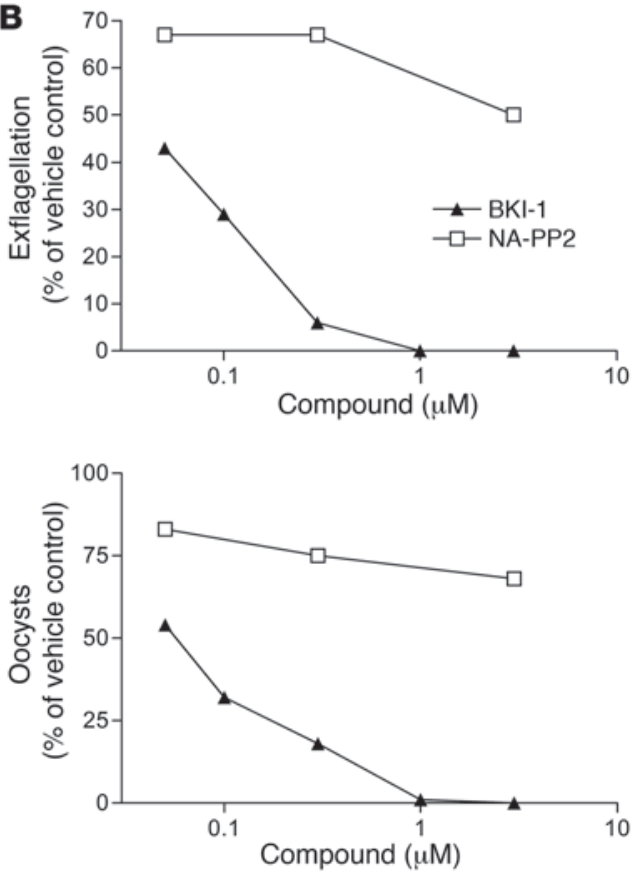
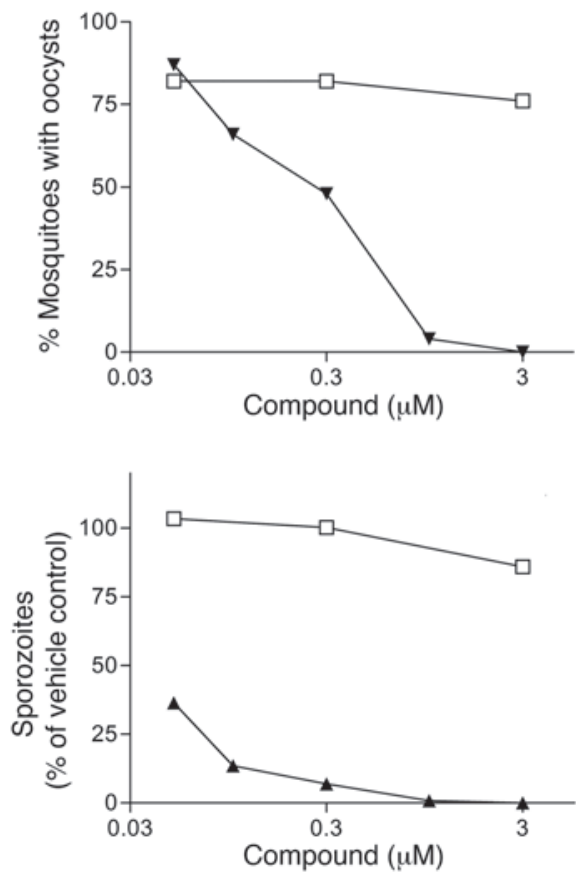

Figure 3

BKI-1 blocks formation of oocysts and thus malaria infection of mosquitoes. (A) Mice were infected with GFPexpressing $P$. berghei and treated with compounds at $3 \mathrm{mg} / \mathrm{kg}$ or 10 $\mathrm{mg} / \mathrm{kg}$ i.p., and 30 minutes later, mosquitoes were fed on mice. Shown are the geometric mean oocyst number on 8-20 dissected mosquito midguts explanted 5 days after feeding. Fluorescence micrographs illustrate typical infection levels in mosquitoes fed on control and BKI-1-treated mice. Scale bar: $500 \mu \mathrm{m}$. Data are representative of 3 experiments. Error bars represent SEM. (B) Compounds were mixed with human blood and aliquots explanted for exflagellation observation, and the blood was fed to $A$. stephensi mosquitoes. A complete suppression of exflagellation was observed with 1 and $3 \mu \mathrm{M} \mathrm{BKI-1}$. Blocking exflagellation with $\mathrm{BKI}-1$ correlated well with the prevention of oocyst and infective sporozoite formation. Sexual stage development in mosquitoes fed with $0.1 \mu \mathrm{M}$ was not completely but was significantly reduced, as shown by more than $86 \%$ in the number of oocyst and infective sporozoites. In a repeat experiment, exflagellation events in the presence of $\mathrm{BKI}-1$ were suppressed completely at 1 or $3 \mu \mathrm{M}$ and more than $90 \%$ at $0.3 \mu \mathrm{M}$. the few salivary glands that were infected was diminished by $86 \%$ relative to untreated controls (Figure 3B). Like other BKIs, this compound is predicted to have little activity against mammalian kinases. In support of this, BKI-1 does not inhibit the kinases SRC and $\mathrm{ABL}$ at $20 \mu \mathrm{M}$, which represent 2 of the most likely off-targets of this compound class (Table 1 ).

Additional evidence for a relationship between $\mathrm{rPfCDPK} 4$ inhibition and blockage of exflagellation, which is consistent with CDPK4 being the target of BKIs (Table 1), is in the absence of atypically small gatekeeper residue in the ATP-binding domains of Plasmodium spp. kinases MAP-2 and SPRK, which are enzymes found to influence the process of exflagellation, and/or NEK-2, NEK-4, GAK, PK7, and CDPK3, found to influence transmission to the mammalian host (13). While there are several FIKK kinases in Plasmodium spp. that contain serine at the gatekeeper position (13), there is no experimental evidence of their involvement in exflagellation or sexual stage developments in mosquitoes.

The active site of Plasmodium vivax CDPK4 is highly homologous to that of PfCDPK4 (Supplemental Figure 3), so that BKI-1 and related compounds should also be useful in blocking vivax malaria. They constitute a class of antimalarial drug candidates that could efficiently block all Plasmodium species infection of mosquito vectors, preventing malaria transmission to humans: a substantial public health implication.

BKI compounds have high selectivity and low toxicity. These compounds did not inhibit proliferation of mammalian cell lines (Table 1) and were not toxic upon administration to mice. BKI-1 has oral bioavailability (Supplemental Figure 4) and can be readily generated from simple starting materials (11), important criteria for drug delivery in economically and infrastructurally challenged malaria endemic regions. A transmission-blocking compound such as BKI-1 could be administered together with drugs active against asexual stages, such as ACT, or otherwise delivered in mass administration campaigns for control.

Emergence of resistance during therapy is a valid concern. However, targeting the sexual stage of malaria is particularly attractive because emergence of resistance is much less likely than when targeting asexual stages. Mosquitoes may ingest up to $10^{4}$ gameto- 
cytes, but usually a much lower number is ingested during blood meals. More often, submicroscopic gametocytemia lead to mosquito infection (14). and only a very small number of parasites are exposed to conditions under which resistance can be selected. Malaria vectors such as Anopheles gambiae effectively block the development of more than $99.99 \%$ of ingested gametocytes, reducing the numbers of gametocytes that infect a mosquito to about 10 to 100 (15). Thus, the probability of few transmitting gametocytes containing a resistance mutation is relatively low in relation to resistance mutations in asexual stages that may have up to $10^{8}$ or $10^{9}$ parasites per infected individual. There seem to be some BKI inhibitory effects on the erythrocytic parasite stage, but this does not track well with their corresponding rPfCDPK4 enzyme inhibition (Table 1). Absence of a significant correlation between rPfCDPK4 inhibition and inhibition of asexual stage parasites suggests that CDPK4 does not play a major role in the asexual part of the Plasmodium life cycle. This provides further evidence of lack of pressure for selection of drug resistance during the asexual erythrocytic stages that could have downstream consequences on prevention of transmission to mosquitoes.

For BKI-1 to completely block transmission, we estimate blood levels of $1 \mu \mathrm{M}$ would need to be maintained for 3 weeks after asexual stage treatment, the length of time gametocytes circulate after asexual stage therapy (16). Extended presence of viable gametocytes in the mammalian host will require prolonged drug bioavailability in the bloodstream for effective transmission blocking to occur. An extensive medicinal chemistry effort is underway to generate a BKI-1 analog that has increased metabolic stability and prolonged exposure. In addition, we have commenced studies to develop a subcutaneous particulate injection formulated with a biodegradable polymer that will ensure slow release and delivery of BKI into the bloodstream over a period of at least 30 days. A combination of this drug treatment with other control strategies will be helpful in accelerating the eradication of malaria.

\section{Methods}

Expression, purification, and enzyme activity assays. Recombinant PfCDPK1 and PfCDPK4 proteins expressed in E. coli (Rosetta Oxford strain) were purified and assayed as described earlier (12). Further details of this and other methods can be found in Supplemental Methods.

1. WHO. World Malaria Report 2010. Geneva, Switzerland: World Health Organization; 2010.

2. Schneider P, et al. (Sub)microscopic Plasmodium falciparum gametocytaemia in Kenyan children after treatment with sulphadoxine-pyrimethamine monotherapy or in combination with artesunate. Int J Parasitol. 2006;36(4):403-408.

3. Bousema T, Drakeley C. Epidemiology and infectivity of Plasmodium falciparum and Plasmodium vivax gametocytes in relation to malaria control and elimination. Clin Microbiol Rev. 2011;24(2):377-410.

4. Billker O, Lourido S, Sibley LD. Calcium-dependent signaling and kinases in apicomplexan parasites. Cell Host Microbe. 2009;5(6):612-622.

5. Nagamune K, Sibley LD. Comparative genomic and phylogenetic analyses of calcium ATPases and calcium-regulated proteins in the apicomplexa. $\mathrm{Mol}$ Biol Evol. 2006;23(8):1613-1627.

6. Kato N, et al. Gene expression signatures and small-molecule compounds link a protein kinase to Plasmodium falciparum motility. Nat Chem Biol.

P. berghei transmission experiments. Mice were injected i.p. with drug or vehicle 3 days after infection. Mice were subsequently anesthetized after 30 minutes and exposed to 25 female A. stephensi mosquitoes. After 5 days, midguts were dissected and imaged using a fluorescence microscope and oocysts quantified.

P. falciparum transmission experiments. P. falciparum strain NF54 parasites were cultured in RPMI 1640 supplemented with $50 \mu \mathrm{M}$ hypoxanthine and $10 \% \mathrm{~A}+$ human serum for 16 days. Cultures were fed to approximately 150 4-day-old A. stephensi mosquitoes. Ten days after feed, approximately 50 mosquitoes from each cage were checked for midgut oocyst infection. On day 14 , remaining mosquitoes in each cage were dissected and pooled to check for salivary gland sporozoites.

Statistics. Error bars represent SEM.

Study approval. All studies were approved by the Institutional Animal Care and Use Committee, Department of Comparative Medicine, University of Washington, and by the Ethical Review Board of Wellcome Trust Sanger Institute.

\section{Acknowledgments}

We thank the following scientists for technical support and valuable conversations: Lynn K. Barrett, Ryan Choi, Wim Hol, Thurston Herricks, Pradip Rathod, Tiffany Silver-Brace, Heather Kain, and Will Betz. This work was supported by NIH grants R01AI089441 and R01AI080625 (to W.C. Van Voorhis) and R01GM086858 (to D.J. Maly); Wellcome Trust WT089085/Z/09/Z; Medical Research Council grant G0501670; and European Commission grant 242095 "EVIMalaR" and 223736 "TransMalariaBloc" (to O. Billker).

Received for publication November 7, 2011, and accepted in revised form March 28, 2012.

Address correspondence to: Wesley C. Van Voorhis, Box 356423 University of Washington, 1959 NE Pacific Ave., Seattle, Washington 98195-6423, USA. Phone: 206.543.2447; Fax: 206.616.4898; E-mail: wesley@uw.edu. Or to: Dustin J. Maly, Box 351700, University of Washington, Seattle, Washington 98195-1700, USA. Phone: 206.543.1653; Fax: 206.685.7002; E-mail: djmaly@uw.edu. Or to: Oliver Billker, Wellcome Trust Sanger Institute, Hinxton, Cambridge CB10 1SA, United Kingdom. Phone: 44.1223.492326; Fax: 44.1223.494919; E-mail: ob4@sanger.ac.uk.

2008;4(6):347-356.

7. Dvorin JD, et al. A plant-like kinase in Plasmodium falciparum regulates parasite egress from erythrocytes. Science. 2010;328(5980):910-912.

8. Billker O, Dechamps S, Tewari R, Wenig G, FrankeFayard B, Brinkmann V. Calcium and a calciumdependent protein kinase regulate gamete formation and mosquito transmission in a malaria parasite. Cell. 2004;117(4):503-514.

9. Kato K, Sudo A, Kobayashi K, Sugi T, Tohya Y, Akashi H. Characterization of Plasmodium falciparum calcium-dependent protein kinase 4. Parasitol Int. 2009;58(4):394-400.

10. Doerig C, Billker O, Pratt D, Endicott J. Protein kinases as targets for antimalarial intervention: Kinomics, structure-based design, transmission-blockade, and targeting host cell enzymes. Biochim Biophys Acta. 2005;1754(1-2):132-150.

11. Murphy RC, et al. Discovery of potent and selective Iinhibitors of calcium-dependent protein kinase 1 (CDPK1) from C. parvum and T. gondii. ACS Med
Chem Lett. 2010;1(7):331-335.

12. Ojo KK, et al. Toxoplasma gondii calcium-dependent protein kinase 1 is a target for selective kinase inhibitors. Nat Struct Mol Biol. 2010;17(5):602-607.

13. Tewari $\mathrm{R}$, et al. The systematic functional analysis of Plasmodium protein kinases identifies essential regulators of mosquito transmission. Cell Host Microbe. 2010;8(4):377-387.

14. Paul RE, Bonnet S, Boudin C, Tchuinkam T, Robert V. Aggregation in malaria parasites places limits on mosquito infection rates. Infect Genet Evol. 2007;7(5):577-586.

15. Vaughan JA, Noden BH, Beier JC. Population dynamics of Plasmodium falciparum sporogony in laboratory-infected Anopheles gambiae. J Parasitol. 1992;78(4):716-724.

16. Eichner M, Diebner HH, Molineaux L, Collins WE, Jeffery GM, Dietz K. Genesis, sequestration and survival of Plasmodium falciparum gametocytes: parameter estimates from fitting a model to malaria therapy data. Trans R Soc Trop Med Hyg. 2001;95(5):497-501. 